\title{
Sul monologo interiore ne 'Les Lauriers sont coupés': strutturazione sintattica e tessitura sonora
}

Laura Santone

\section{(2) OpenEdition}

1 Journals

\section{Edizione digitale}

URL: http://journals.openedition.org/studifrancesi/32682

DOI: 10.4000/studifrancesi.32682

ISSN: 2421-5856

\section{Editore}

Rosenberg \& Sellier

\section{Edizione cartacea}

Data di pubblicazione: 1 décembre 2005

Paginazione: 542-551

ISSN: 0039-2944

\section{Notizia bibliografica digitale}

Laura Santone, «Sul monologo interiore ne 'Les Lauriers sont coupés': strutturazione sintattica e tessitura sonora», Studi Francesi [Online], 147 (XLX | III) | 2005, online dal 30 novembre 2015, consultato il 20 avril 2021. URL: http://journals.openedition.org/studifrancesi/32682 ; DOI: https:// doi.org/10.4000/studifrancesi.32682

\section{(c) (i) $\odot$}

Studi Francesi è distribuita con Licenza Creative Commons Attribuzione - Non commerciale - Non opere derivate 4.0 Internazionale. 


\section{Sul monologo interiore \\ ne 'Les Lauriers sont coupés': \\ strutturazione sintattica e tessitura sonora}

Le osservazioni che seguono traggono impulso dalla lettura delle numerose pagine che indagano la tecnica del monologo interiore ${ }^{1}$, in particolare muovono da quei contributi critici che in una prospettiva di universi paralleli - eminentemente il testo joyciano e, talora, l'esercizio creativo di Valery Larbaud ${ }^{2}$ - intersecano l'organizzazione testuale di Les Lauriers sont coupés, il breve romanzo che Edouard Dujardin pubblica nel $1887^{3}$.

Accade di sovente, all'interno di tale campo prospettico, che la complessità del testo joyciano - segnatamente l'Ulysses - catalizzi un'attenzione più marcata, vocata a disperdere o a lasciare in ombra le caratteristiche più forti del testo di Dujardin. I punti di emergenza di Les Lauriers sont coupés perdono così in tensione, da cui l'effetto di recepirli quale "testo secondo" cui l'Ulysses fungerebbe da supporto, laddove occorre invece ribaltare il rapporto avendo Dujardin fornito, come è peraltro lo stesso Joyce a rivelare, gli elementi portanti della nuova tecnica narrativa ${ }^{4}$.

La presente analisi, pertanto, si prefiggerà rispetto a Les Lauriers sont coupés una circoscrizione di campo, il che equivale a dire l'attraversamento del testo nella sua specificità, nella messa a fuoco di quel materiale verbale che amalgama il piano enunciazionale nei suoi livelli di significato e nella sua attività di espansione. Rispetto a Ulysses una tale operazione è esplicitazione supplementare delle nervature della scrittura joyciana, fermo restando in quest'ultima il dispositivo dell'invenzione vera e propria.

\section{Strutturazione sintattica}

In una lettera che l'8 aprile 1888 Mallarmé inviava a Dujardin, all'indomani della pubblicazione in volume di Les Lauriers sont coupés, si legge:

Lei sa che ho particolarmente apprezzato questo romanzo fin dalla prima lettura sulla «Revue Indépendante»; e oggi, sfogliandolo, mi accorgo che in esso lei ha definito un modo $d i$ notazione piroettante e rapido ${ }^{5} .$.

(1) Si rimanda, per una visione d'insieme, alla bibliografia annessa al mio Voci dall'abisso. Nuovi elementi sulla genesi del monologo interiore, Bari, Edipuglia, 1999.

(2) Con particolare riferimento ai due romanzi Amants, beureux amants (1921) e Mon plus secret conseil (1923).

(3) Il romanzo era dapprima apparso a puntate su «La Revue Indépendante», dal maggio all'agosto 1887. L'edizione in volume uscì solo l'anno successivo, presso la casa della rivista stessa. Seguì, nel 1897, una ripubblicazione per le edizioni Mercure de France e nel 1924 un'edizione riveduta per la casa editrice Messein, accompagnata da una prefazione di Valery Larbaud.

(4) Si veda quanto E. Dujardin riferisce in pro- posito in Le Monologue intérieur, son apparition, ses origines, sa place dans l'œuvre de James Joyce, Paris, Messein, 1931. Trad. it. Il monologo interiore, con una introduzione di J. Risset, Parma, Pratiche Editrice, 1991. Chiarificatrice risulta peraltro, a riguardo, la dedica che lo stesso Joyce appose nel 1929 sulla copia della traduzione francese dello Ulysses inviata in omaggio a Dujardin: «A E.D. Annonciateur de la parole intérieure, le larron impénitent, J.J.» (lettera del 22 novembre 1929 a Harriet Shaw Weaver, in J. Joyce, Lettere, a cura di G. Melchiori, Milano, Arnoldo Mondadori Editore, 1974 , p. 500).

(5) E. Dujardin, Il monologo interiore, op. cit., p. 25, corsivo mio. 
Ma quali forme assume nella messa in atto del récit questo «modo di notazione piroettante e rapido»? Quali le sue marche sul piano della costruzione sintattica? Quali i suoi effetti di senso? Interrogativi che delimitano, a loro volta, un obiettivo, e sulla scia dei quali si organizzeranno i dati che via via emergeranno dall'indagine.

\section{Pluralizzazione della persona verbale: «je», «nous».}

Luogo del pensiero intimo in formazione, il monologo interiore orienta l'attenzione del lettore verso quel centro focale che Joyce individuava, percorrendo Les Lauriers sont coupés, ne «lo svolgimento ininterrotto dei pensieri del personaggio principale ${ }^{6}$ : abbozzi di frasi ridotte al minimo sintattico, articolate in divenire, secondo le linee di fuga della soggettività in azione. Deflagrazione del modello narrativo tradizionale, il monologo interiore corrode altresì lo statuto tradizionale della comunicazione in quanto, come evidenziano nella loro mirabile analisi di alcuni frammenti di Ulysses Paola Pugliatti e Romana Zacchi, esso «compie l'azione del tutto peculiare di autocomunicare» ${ }^{7}$. E, di fatto, Daniel Prince, il giovane protagonista dei Lauriers sont coupés, non si rivolge ad altri se non a se stesso - come più tardi faranno Bloom, Stephen, Molly -, e «quando si parla a se stessi», continuano le due autrici, «di solito non si ordina, non si promette, non si rimprovera, non si persuade, non si accusa» ${ }^{8}$. L'istanza monologante, cioè, esula dai verbi performativi per saldarsi intorno ai cosiddetti verbi creatori di mondi: "penso", "immagino", "sogno", "desidero", "fantastico”, ecc.

Data questa premessa, si osservino ora i seguenti enunciati:

Un soir de soleil couchant, d'air lointain, de cieux profonds; [...] dans l'illusion des choses qui s'engendrent et qui s'enfantent, ... je surgis...

Voici le consommé, le consommé fumant; attention à ce que le garçon ne m’en éclabousse rien. Non, mangeons. Ce bouillon est trop chaud; essayons encore ${ }^{10}$.

Revenons à la question; je veux m'amuser à songer comment j'arrangerais les choses, si je devenais riche; oui, arrangeons cela, tout en marchant ${ }^{11}$.

Adieu, adieu, partons. L'escalier sera éclairé par le gaz; j'ouvre la porte; j'éteins les bougies; voilà; ne heurtons rien; la porte refermée; descendons; mes gants; ils sont propres, qui, convenables ${ }^{12}$.

Daniel Prince pensa ed enuncia i propri pensieri, sorge dalle proprie fantasticherie, e organizza - crea - il proprio universo di discorso attraverso un "io" che copre, al presente, l'intera prospettiva enunciazionale. Ma si tratta, e gli esempi lo segnalano chiaramente, di un "io" complesso, che assume su di sé l'amplificazione che gli viene dalla sovrapposizione della prima persona plurale. Un "io" massiccio, che conferisce tratti ancora più rarefatti al flusso della coscienza, e nel quale è già possibile ricono-

(6) Ibid. p. 24.

(7) P. Pugliatti-R. Zacchi, Terribilia meditans, Bologna, Il Mulino, 1983, p. 122. Corsivo del testo.

(8) Ibid.

(9) E. Dujardin, Les Lauriers sont coupés, Paris,
Le Dilettante, 1989, p. 9. Segnaliamo sin da ora che $i$ corsivi delle citazioni, salvo rinvio al testo, sono da intendersi nostri.

(10) Ibid., p. 21.

(11) Ibid., p. 32.

(12) Ibid., pp. 67-68. 
scere quell'“io" dilatato quale sarà descritto più tardi da Emile Benveniste: 1' "io" proiettantesi «oltre la persona in senso stretto, accresciuto e nello stesso tempo con dei contorni vaghi» ${ }^{13}$.

Sul polo del lettore, tale contaminazione tra l'“io" e il "noi" rafforza l'illusione di coincidenza - di «embrayage» secondo la terminologia di Greimas - con il soggetto enunciante. In quanto la pluralizzazione della prima persona singolare sfocia, come insegna sempre Benveniste, «in un'espressione più larga e diffusa: è il "noi" dell'autore e dell'oratore» ${ }^{14}$, assimilabili all'interno di una visione autoreferenziale in cui l'informazione - intesa quale distribuzione gerarchica di un significato - non si dà più prioritaria e ove il linguaggio postula, lacanianamente, la separazione del soggetto nella sua relazione d'oggetto, ovvero la struttura del «manque»»

\section{Le forme temporali del verbo: presente, imperativo, infinito}

Il monologo interiore non accusa la «necessità di dire il tempo, poiché il suo tempo è quello che occorre per la sua enunciazione» ${ }^{16}$. Sicché il monologante esorbita dal piano storico vivendo nell'istante, il cui incessante divenire rappresenta l'evento; nessuna memoria storica lo contiene, poichè l'enunciazione storica verrebbe a escludere il presente, intralciando pesantemente il vivo lavoro dell'bic et nunc ${ }^{17}$.

Ora, osservando attentamente il registro verbale di Les Lauriers sont coupés si nota che Dujardin, unitamente al presente, fa spesso ricorso alle forme verbali dell'infinito e dell'imperativo. In più luoghi testuali si tratta di un uso congiunto, poiché i due tempi si accavallano, come a voler connotare con più efficacia il carattere sorgivo dell'io monologante. Non si danno, pertanto, quali demarcatori di una temporalità specifica: e se tale funzione meglio riesce, per sua natura, all'infinito ${ }^{18}$, nondimeno si può asserire dell'imperativo, la cui esortazione all'azione non è affatto riducibile alla struttura del rinvio, vale a dire al compimento dell'azione stessa, bensì alla semplice trascrizione del gesto "pensato", "desiderato", "fantasticato" - ritornano i verbi creatori di mondi -, il che colloca, ancora una volta, il processo dell'enunciazione all'interno di una situazione autocomunicativa.

Si vedano a titolo esemplificativo le sequenze scelte:

- Comment? partir? - Oui, laissez, abandonnez ceci; au centuple vous le retrouverez; tous deux, sauvons-nous, partons, venons-nous en! Et je la prendrais dans mes bras ${ }^{19} \ldots$

Aimer; et honorer son amour, respecter son amour, aimer son amour ${ }^{20}$.

Se marier; épouser une femme aimée; pouvoir épouser une femme qu'on aime: l'avoir. On trouverait donc ces choses, se marier, être ensemble, avoir sa femme ${ }^{21}$.

(13) E. Benveniste, Problèmes de linguistique générale, Paris, Gallimard, 1966; trad. it. Problemi di linguistica generale, Milano, Il Saggiatore, 1971, p. 280.

(14) Ibid. 365.

(15) Cfr. J. Lacan, Ecrits, Paris, Seuil, 1966, p.

(16) P. Pugliatti-R. Zacchi, op. cit., p. 117; corsivo del testo.

(17) Fa rilevare in proposito Benveniste: «Lo storico non dirà mai io, né $t u$, né qui, né ora...»; e ancora: «Il presente è escluso, eccettuato il caso rarissimo di un presente atemporale come il "pre- sente di definizione”». Cfr. op. cit., p. 285.

(18) Interessante è ricordare a riguardo quanto osserva Enrico Arcaini sulla natura dell'infinito: «In quanto verbo rivela soltanto un'idea piuttosto vaga di processo, perché non possiede marche tali da collocarlo in una categoria la cui funzione primaria è di situare l'evento». Cfr. E. ArCaINI, Italiano $e$ francese. Un'analisi comparativa, Torino, Paravia Scriptorium, 2000, p. 246.

(19) E. Dujardin, Les Lauries sont coupé, op. cit., p. 32

(20) Ibid., p. 14, corsivo del testo.

(21) Ibid., pp. 36-37. 
Puisque je n'ai rien à faire, examinons un peu, mais sérieusement, comment je dois agir ce soir chez Léa: évidemment, demeurer avec elle jusqu'à minuit ou une heure, puis m’en aller... ${ }^{22}$

\section{La frase nominale}

La frase nominale rappresenta uno dei più forti indici linguistici atti a connotare una situazione di autocomunicazione. Essa, infatti, pone l'enunciato al di fuori dello spazio assertivo riempito dal verbo, ignorando e la «funzione coesiva», ovvero l'organizzazione degli elementi dell'enunciato in una struttura completa, e la «funzione assertiva», che dà all'enunciato un predicato di realtà ${ }^{23}$. Atemporale, impersonale, non modale, all'asserzione nominale non è riconosciuto di mettere in rapporto «il tempo dell'evento con il tempo del discorso sull'evento»: poggerà pertanto su un termine «ridotto al suo esclusivo contenuto semantico» ${ }^{24}$.

Esaminando in tale prospettiva il testo di Dujardin, salta subito all'occhio una particolare costruzione della frase nominale, concepita per accumulazione all'interno di blocchi di enunciato che si danno come luoghi di estrema condensazione, ma altresì come "buchi" del sistema significante. Configurano, infatti, frasi senza sviluppo, "inorganiche", che annotano una sintassi segmentata, per detractio - le «frasi brevi, vivaci e leggere» di cui riferirà Edmond Jaloux ${ }^{25}$-; frasi la cui enumerazione sembra procedere secondo i principi di un arpeggio musicale: una successione di pizzicato - le impressioni di Daniel Prince -, suscettibile di sviluppare un effetto di molteplice nel simultaneo - la molteplicità/simultaneità degli stati di coscienza. Ė quanto accade nel passo selezionato:

La rue, noire, et la double ligne montante, décroissante, du gaz; la rue sans passants; le pavé sonore, blanc sous la blancheur du ciel clair et de la lune; au fond, la lune, dans le ciel; le quartier allongé de la lune blanche, blanc; et de chaque côté, les éternelles maisons; muettes, grandes, en hautes fenêtres noircies, en portes fermées de fer, les maisons; dans ces maisons, des gens? non, le silence; [...] là-bas, tout au là-bas, une plus grande clarté, le boulevard Malesherbes, des feux rouges et jaunes, des voitures, des voitures et de fiers chevaux; immobilement, au travers des rues, dans une calme immobile, des voitures... ${ }^{26}$

\section{Anteposizione e disgiunzione}

Un altro aspetto caratterizzante la distribuzione sintattica del «tout venant» nel testo di Dujardin è l'impiego dell'aggettivo anteposto al soggetto cui si riferisce. È una soluzione che si presta all'emersione subitanea di un'impressione centrale, la quale si fa pretesto per un processo di associazioni:

Illuminé, doré, rouge, avec les glaces, cet étincellement; quoi? le café; le café où je suis ${ }^{27}$.

Excellent serait ce petit morceau blanc, avec les raies qu'ont marqué les arêtes. Tant pis; je ne le mangerai pas; je m'essuie les doigts avec ma serviette; un peu rude, ma serviette ... Vide est la banquette en face... ${ }^{28}$

(22) Ibid., p. 51

(23) E. BENVEniste, op. cit., p. 182.

(24) Ibid., p. 187.

(25) Cit. in E. Dujardin, Il monologo interiore, op. cit., p. 41.
(26) E. Dujardin, Les Lauries sont coupé, op. cit., p. 32

(27) Ibid., p. 20.

(28) Ibid., p. 23. 
E dove evidente risulta il verificarsi, in parallelo, della disgiunzione del lessemasoggetto dalla sua regolare disposizione sintattica; risultato che realizza, dal punto di vista semantico, una sospensione del senso e dei rapporti di coerenza con gli enunciati contigui, dando il massimo rilievo alla percezione individuale.

\section{Interpunzione}

«Una sorprendente musica di punti e virgole»: venivano così definiti Les Lauriers sont coupés da George Moore ${ }^{29}$ in una lettera scritta a Dujardin il 17 maggio 1887 mentre il romanzo usciva a puntate su «La Revue Indépendante».

Dalle citazioni sinora esaminate, del resto, è già possibile constatare, a un primo livello, come la punteggiatura esuli dall'uso tradizionale e contrassegni un incessante lavoro di tessitura dell'enunciato nella direzione della pluralità, dell'eterogeneità, della mobilità. Ma per descriverne più approfonditamente usi e funzioni si prenderà spunto da uno dei luoghi testuali in cui l'io monologante si fa più vicino all'area dell'inconscio, ovvero il momento in cui Daniel Prince, alle soglie del sonno, è in quello stato di dormiveglia che sfuma e dissolve percezioni e pensieri:

La voilà immobile, si finement jolie, si jeunement coquette; oh! la triste existence qu'est la sienne; à celui qui l'aime, quel amour il faut, pour lui adoucir les amertumes; pauvre qui va, à vingt ans, livrée aux mauvaises heures... ensemble, au contraire, ainsi dormir, en l' oubli... [...] «j' t'aim' mieux qu'mes moutons»... j' t'aim' mieux... cette fille, yeux éhontés, frêle, aux lèvres rouges... la chambre, la cheminée haute... la salle... mon père... tous trois assis, mon père, ma mère... moi-même... pourquoi ma mère est-elle pale?... elle me regarde... nous allons dîner, oui, sous le bosquet... la bonne... apportez la table... Léa... [...] tout scintille... vous riezvous?... les becs de gaz s'alignant infiniment... oh!... la nuit... froide et glacée, la nuit... Ah!!! mille épouvantements... quoi? on me pousse, on m'arrache, on me tue... Rien... un rire... la chambre... Léa... Sapristi... m'étais-je endormi? ${ }^{30}$.

Il primo rilievo va fatto sul polo del lettore: i segni grafici di interpunzione non forniscono alcuna istruzione di lettura, in quanto non istituiscono legami sintattici né relazioni di consecuzione semantica, aprendo unicamente su una molteplicità di stati mentali. Postulano, di conseguenza, un piano autoreferenziale - il piano della coscienza -, che non inferisce vincoli logico-temporali.

Il secondo rilievo implica una focalizzazione più netta del passo registrato, nella considerazione delle funzioni assunte dai segni interpuntivi ivi operanti. Anzitutto la profusione dei punti di sospensione, da leggersi in diretto rapporto con la mancanza di un punto fermo. Sono espedienti che, se da un lato mirano a ricreare l'inafferrabilità delle percezioni, dall'altro ne predicano l'indecidibilità. Non a caso l'effetto prodotto è quello di creare stacchi spostando continuamente l'attenzione su una sequenza di segmenti che si offrono come tagli - buchi - del discorso.

E vuoti semantici circoscrivono altresì:

- la virgola (,), il cui impiego realizza, più che una funzione sommativa, un legame di contemporaneità;

- il punto e virgola (;), che sfuma o elude totalmente le connessioni tra le sequenze intercalate;

- il punto interrogativo (?), che disattende l'aspettativa della risposta;

- il punto esclamativo (!), che si moltiplica talora "per irradiazione" - (!!!) -, 
senza chiudere il percorso di una sequenza compiuta, ma assolvendo unicamente all'espansione della misura ritmica dell'enunciato.

Da segnalare è infine, per tutta l'estensione del romanzo, l'assenza del segno grafico dei due punti - salvo quando esso debba introdurre il discorso diretto -, demarcativo dell'istanza di specificazione, del cosiddetto valore cataforico. È allora chiaro come il monologo interiore sia teso a disattendere, nel suo complesso, ogni previsione di specificazione, spostando invece di continuo l'attenzione del lettore sul linguaggio in quanto processo in atto di evoluzione e di dissoluzione, designando così, attraverso un gioco di esitazioni, il luogo di applicazione dell'operazione poetica, là dove il messaggio, strappato «dai cardini della logica» ${ }^{31}$, è sempre portatore di un tasso di ambiguità, poiché, come hanno sottolineato gli studi di Jakobson, «l'equivalenza è promossa al grado di elemento costitutivo della sequenza» ${ }^{32}$.

Dopo aver puntualizzato, pertanto, taluni aspetti inerenti alla strutturazione sintattica di Les Lauriers sont coupés, si tenterà ora di entrare nel contesto fonetico delle sequenze, cercando di captarne le vibrazioni eufoniche e ritmiche; o ancora, si tratterà di scivolare in quell'alone in cui mormora, per dirla con Michel Foucault, il «sordo lavoro delle parole» ${ }^{33}$, ovvero il tempo in battere e in levare del flusso di coscienza.

\section{Tessitura sonora}

Anche se letti secondo il ritmo del discorso verbale piano Les Lauriers sont coupés lasciano affiorare frammenti metrici organizzati secondo un gioco di iterazioni timbriche orizzontali - allitterazioni, assonanze, consonanze - e verticali - rime - che restituiscono l'organizzazione del testo in effetti a-significanti molto vicini a quelli dell'arte musicale.

Del resto, nelle intenzioni di Dujardin, il monologo interiore doveva esattamente realizzare il «ritorno alle origini musicali del linguaggio» e contrassegnare così «il folgorante ingresso della poesia del romanzo» ${ }^{34}$. Senza voler qui ritornare sulla nota influenza esercitata in tale direzione dalla musica di Wagner, va tuttavia ricordato che l'ascolto a Bayreuth, nel 1884, della Tetralogia, fortemente impregnata di quelle frasi brevi, senza sviluppo, chiamate Leitmotive, indurranno circa tre anni dopo Dujardin a concepire la sua prova di romanziere in una pratica di riverberi e di echeggiamenti interni vocati a trasporre nell'organizzazione linguistica del testo il principio del suono. Testimonianza di questo procedimento forniscono in particolare due sequenze delle quali la prima, collocata alla fine del secondo capitolo, illustra nella concentrazione accordata alla rima il primato dell'omofonia sul tasso d'informazione; mentre la seconda, collocata poco prima della fine del capitolo terzo, risegmenta lo spazio

(31) Ibid., p. 44: «Si tratta ora di rompere la cornice, di strappare la frase (Prendi l'eloquenza e torcile il collo!) dai cardini della logica, di liberare le parole dalle forme della sintassi e associarle in un ordine spontaneo, oppure di non associarle affatto». Così scriveva Louis Gillet richiamando l'attenzione sulle caratteristiche del monologo interiore.

(32) R. JAKOBSON, Essais de linguistique générale, Paris, Editions de Minuit, 1963; trad. it. Saggi di linguistica generale, Milano, Feltrinelli, 1966, p. 192. E sempre in riferimento alla funzione poetica: «Il predominio della funzione poetica rispetto a quella referenziale non annulla il riferimento, ma lo rende ambiguo» (p. 209).

(33) Riprendiamo qui la suggestiva espressione che dà il titolo al capitolo IV di Ceci n'est pas une pipe, Paris, Fata Morgana, 1986.

(34) Non si può non ricordare, in proposito, la replica di Dujardin a Gabriel Marcel che lo accusava, sulla «Nouvelle Revue Française» del febbraio 1925 , di aver voluto trasporre nel romanzo i procedimenti della poesia e della musica: «Ahimè, caro signore, era proprio quello che volevo!» (Il monologo interiore, op. cit, p. 50). Dujardin, del resto, già in occasione di alcune conferenze tenute in Germania prim'ancora che uscisse il suo saggio sul monologo interiore aveva affermato che «l'origine del linguaggio non è il gesto ma il grido, vale a dire la musica» (cit. da E. VAN DER STAAY, Le Monologue intérieur dans l'ouvre de Valery Larbaud, Paris, Genève, Champion-Slatkin, 1987, p. 179). 
semantico in un sottile reticolo di cellule ritmiche che svelano del testo il suo compatto coefficiente poetico.

Si osservi attentamente la prima delle due sequenze prese in esame:

Que c'est agaçant, toujours diner au restaurant! personne ici à qui parler; personne à voir; pas une femme à regarder; depuis huit jours, pas une femme; un tas de messieurs quarts de chic; ils viennent ici par gueuserie; des décavés; puis des avoués de province qui se croient chez Bignon ${ }^{35}$.

Come si può constatare, la rima opera quale principio costitutivo dell'intero passo, articolando una tessitura timbrica che si espande per attrazione omofonica facendo slittare la percezione del lettore dal referenziale verso l'udibile. Ma vi è di più. Essa si rivela sollecitare, accanto a un parallelismo nel ritmo - ricorrenza di sillabe -, un parallelismo nel metro, rintracciabile nella successione delle unità metriche che si ottengono trascrivendo la sequenza in "versi":

Que c'est agaçant,

$$
5
$$

toujours diner au restaurant!

8

personne ici à qui parler;

8

personne a voir;

pas une femme à regarder;

8

depuis huit jours,

4

pas une femme;

4

un tas de messieurs quarts de chic;

8

ils viennent ici par gueuserie;

8

des décavés;

4

puis, des avoués de province

8

qui se croient chez Bignon

6

Su dodici «versi» l'ottonario risulta essere la misura ritmica di base, ricorrendo ben sei volte e istituendo pertanto un perfetto parallelismo esplicitabile nel rapporto 1 su 2. Altrettanto è possibile verificare nell'occorrenza del quartenario, che nell'economia dell'intera sequenza attua una distribuzione parallela di 1 su 3.

Si passi ora alla seconda sequenza:

[...] ô mon ami au sourire et au rire léger, aux yeux qui rient, aux grands yeux, petite rieuse bouche, oiu, souriantes lèvres! dans l'ombre gisent les confus jardins, sous le ciel clair, et c'est sa jolie tête blonde, moqueuse et petitement juvénile, fin nez, mignonne face, fins blonds cheveux, blanche fine peau, enfant qui sourit et me rit et me moque et nous nous chérissons;

(35) E. Dujardin, Les Lauriers sont coupés, op. cit., pp. 27-28. Dove i corsivi sono ovviamente tesi

a mettere in risalto i giochi di rima. 
dans cette nuit, sur le balcon fuyant, sur l'indistinct des murs lointains, dans l'air tiède et nocturne, parmi l'alentour qui s'efface, tu es belle et tu es gracieuse; gracieuse divinement, tu marches, en le bercement de tes hanches, et tu marches mollement, sur le tapis, auprès de la table où sont les fleurs, en ton exquis jaune salon; au long des fleurs sur le tapis moiré tu marches mollement, inclinant ta tête à droite lentement, à gauche lentement, avec des sourires blancs, face éburine aux foux cheveux, souriante, lentement, ondulante, tu passes, tu passes, tu marches... ${ }^{36}$

A prodursi è uno straordinario effetto di strumentazione fonica. L'aggregazione della massa verbale, ora molle ora dura, ora squillante ora profonda, persegue una composizione di incroci che attraversano la superficie del testo eludendo la linea - il tracciato orizzontale del cosiddetto asse sintagmatico - e inanellando al suo posto le maglie di un vero e proprio reticolo. Se ne osservi la configurazione.

La rima. Visibilmente intessuta sulla pressione della rima, la sequenza selezionata ne potenzia l'insistenza attraverso nessi allitterativi che si coniugano a forti effetti di rima ribattuta, ovvero la rima il cui corpo fonico si dà «per sottrazione di una o più lettere del vocabolo maggiore» ${ }^{37}$ :

ô mon amie au sourire et au rire lèger, aux yeux qui rient;

enfant qui sourit et me rit et me moque et nous nous chérissons ${ }^{38}$;

Ma è opportuno, a questo proposito, un altro rilievo. Occorre cioè aggiungere che dietro la rima ribattuta è reperibile un secondo dispositivo, adibito a realizzare, accanto all'equivalenza eufonica, un effetto visivo: il triangolo magico. Se trascritte in linea verticale, infatti, le rime sopra evidenziate danno:

$\begin{array}{cc}\text { sourire } & \text { sourit } \\ \text { rire } & \text { rit } \\ \text { [ri] } & \text { ché[ri] ssons }\end{array}$

Trasmigrazione «iconica» della scrittura, fondazione non lineare del senso, sospensione dell'istanza di enunciazione con parallelo effetto di decentramento del soggetto enunciante ${ }^{39}$. L'equivalenza del suono contrassegna i punti di fuga della messain-discorso, marcando la priorità del significante sul significato ${ }^{40}$ : «mezzi nuovi», «appropriati alle circostanze del nuovo linguaggio», al fine di ricrearne $\ll$ l'antico potere» ${ }^{41}$.

Anadiplosi e versi serpentini. Altra caratteristica saliente della sequenza scelta è una costellazione di lessemi regolarmente reiterati che si sovrappone, quasi contro-

(36) Ibid., pp. 45-46.

(37) Cfr. G. Pozzi, Poesia per gioco, Bologna, Il Mulino, 1984, p. 94.

(38) Nel vocabolo "chérissons" la rima ribattuta è interna, interessando l'unità sillabica /ri/.

(39) Interessante risulta, in questa prospettiva, la seguente osservazione di Stefano Ágosti circa l'esperienza poetica: «Nel poetico, la struttura del discorso vero e proprio risulta occultata (o accantonata o sospesa), con effetto parallelo di sospensione del Soggetto dell'enunciazione, il quale figura esso stesso come un elemento, alla stregua di tutti gli altri, delle strutture in atto del paradigma». In Critica della testualità, Bologna, Il Mulino, 1994, p. 120.

(40) Secondo J. M. Lotman nei rapporti di relazione che vengono a istituirsi all'interno di un testo poetico la coincidenza del suono, nella fattispecie la rima, riduce la possibilità di una distribuzione gerarchica dell'informazione. La rima, spiega Lotman, «è una coincidenza di parole o di loro parti, con marcata non coincidenza di significato» ( $\mathrm{La}$ struttura del testo poetico, Milano, Mursia, 1976, p. 151).

(41) Cfr. E. Dujardin, Il monologo interiore, op. cit., p. 48. 
bilanciandola, a una densa stratificazione di assonanze e consonanze. Se ne ricava un effetto di scorrimento e di accavallamento insieme, che connota poeticamente - e musicalmente - il tempo del discorso ${ }^{42}$.

Ma all'interno di tale costellazione, nella distribuzione data alle unità di significazione, opera un piano più nascosto, ove sono riconoscibili due artifici dichiaratamente poetici, tipici della strofe medievale: l'anadiplosi, vale a dire la ripetizione del vocabolo finale all'inizio del segmento seguente, e i versi serpentini, che si ottengono quando a essere ripetuto negli estremi opposti è un segmento linguistico più este$\mathrm{so}^{43}$.

Si veda l'effetto anadiplosi: marches...

tu es belle et tu es gracieuse; gracieuse divinement... ondulante, tu passes; tu passes, tu

cui si intreccia l'effetto versi serpentini:

et tu marches mollement $[\ldots]$

au long des fleurs sur le tapis moiré tu marches mollement,

inclinant ta tête à droite lentement, à gauche lentement,

avec des sourires blancs, tace éburine aux foux cheveux,

souriante, lentement,

ondulante, tu passes, tu marches...

A essere in gioco è, nell'intera sequenza, il principio della contaminazione fonica, che istituisce un parallelismo attraverso il quale il suono arriva, all'orecchio del lettore, come un'esitazione del senso.

Ricorrenza del fonema [ã]. La sequenza esaminata manifesta, infine, una forte concentrazione del fonema [ã]. Esso compare, nei seguenti lessemi:

/grands/, /souriantes/, /petitement/, /blanche/, / enfant/, /fuyant/, /alentour/, /divinement/, /bercement/, /hanches/, /mollement/ (frequenza: 2), /lentement/ (frequenza: 3), /inclinant/, /blancs/,/souriante/, /ondulante/;

e nelle preposizioni:

/en/, /dans/ (frequenza: 3).

La ricorrenza di tale fonema è talmente massiccia da dare l'impressione di costituire la cifra della tessitura fonico-timbrica dell'intera sequenza. La sua distribuzione si esplicita in una vera e propria disseminazione strutturante la superficie testuale in un tessuto di piccole molecole che scorrono le une sulle altre creando un effetto di fluidità - e di conseguente disgiunzione - delle unità di significato. Tale fonema, poi, si presta a un'osservazione supplementare. Se considerato in rapporto a tutto il testo dei Lauriers sont coupés esso si lascia cogliere come la marca fonica costitutiva del flusso di coscienza (e forte è la tentazione, in questo caso, di individuare nell'organizzazione complessiva del testo quelle che potrebbero definirsi le sue "isotopie fonemiche”). Il fonema [ã] connota cioè, in ricorrenza e in dominanza ${ }^{44}$, lo scorrere - e la

(42) Preziosa è in senso un'osservazione che, ancora una volta, ci viene da Jakobson: «Il ritmo delle sequenze è un procedimento che, al di fuori della funzione poetica, non trova applicazione nel linguaggio. Soltanto nella poesia, con la sua reiterazione regolare di unità equivalenti, si avverte il tempo del flusso parlato, come accade per il tempo musicale», op. cit., p. 192.

(43) Cfr. G. Pozzi, op. cit., p. 113.

(44) Esattamente come si addice a una vera isotopia connotativa, secondo la concezione che ci viene da Greimas, il quale, peraltro, è stato il primo a postulare la rintracciabilità di un livello fonemico suscettibile di dare, sul piano dell'organizzazione 
dissoluzione - di parole fluide: i bagliori della Parigi di una sera «de soleil couchant», «des espaces infiniment étendus», «dans l'illusion des choses qui s'engendrent et s'enfantent»... ${ }^{45}$

$$
* * *
$$

Attraverso due percorsi intrecciati l'uno sull'altro, abbiamo voluto far emergere le caratteristiche forti della tecnica del monologo interiore quale Dujardin la applica nei Lauriers sont coupés, sebbene essa, raffrontata al testo joyciano, costituisca, come rileva giustamente in proposito Jacqueline Risset, una «intuizione basilare» ${ }^{46}$. Un' intuizione da sempre esplicitata nel raffronto con Ulysses, ma suscettibile, come si è visto di volta in volta negli esempi esaminati, di portare alla luce i suoi effetti più fecondi se chiamata a confrontarsi con la tipologia fondante il discorso poetico. Assunto "poeticamente", infatti, il romanzo di Dujardin svela allo sguardo del critico - e del lettore - luoghi testuali di inedita bellezza, ove il materiale linguistico, finemente organizzato, offre la manifestazione in re di quello che sarà successivamente, nella sua straordinaria e impareggiabile partitura, il monologo interiore joyciano.

Un'ultima osservazione. In una lettera scritta il 29 febbraio 1924 a René Lalou, Valery Larbaud parlava di «monologo senza voce della coscienza», definendolo «non declamabile» e aggiungendo che lo si poteva leggere solo «con un tono il più vicino possibile alle parole pronunciate in sogno» ${ }^{47}$. Analogamente, Edmond Jaloux parlava di una tecnica «che tesse e disfa ininterrottamente tele quasi indistinte nel fondo della nostra anima $\gg^{48}$. Il monologo interiore, cioè, sotto la marca del "silenzio" - il sogno, l'anima -, veniva precisato nella sua vicinanza con l'inconscio. Ed è proprio tale vicinanza a chiarire, a conclusione della presente analisi, il luogo esatto ove esso si situa: oltre la soglia della pertinenza linguistica, là dove le parole, percepite «indipendentemente dalla sequenza ordinaria», «sono ciò che non si dice discorso» ${ }^{49}$.

LAURA SANTONE

espressiva, una lettura isotopa. Si rimanda a: $D u$ sens, Paris, Seuil, 1970, trad. it. Del senso, Milano, Bompiani, 1974.

(45) E. Dujardin, Les Lauriers sont coupés, op. cit., p. 9.

(46) J. RISSET, «... l'instant pris à la gorge», introduzione a E. Dujardin, Il monologo interiore, op. cit., pp. 7-18.

(47) Ibid., p. 39.

(48) Ibid., p. 41.

(49) $\mathrm{Ci}$ è parso qui significativo - sia per l'influenza che la dottrina simbolista ha esercitato su Dujardin, sia per la centralità che la lingua ha rivestito nella riflessione mallarmeana - raccogliere l'eco proveniente da Le Mystère dans les Lettres di Mallarmé. Il passo cui alludiamo è il seguente: «Les mots, s'exaltent à mainte facette reconnue la plus rare ou valant pour l'esprit, centre de suspens vibratoire: qui les perçoit indépendamment de la suite ordinaire, projétés, en parois de grotte, tant que dure leur mobilité ou principe, étant ce qui ne se dit pas du discours» (S. MALlaRMÉ, Euvres complètes, Paris, Gallimard, Bibliothèque de la Pléiade, 1945, p. 386). Nondimeno significativa riteniamo una suggestione che ci viene da uno stimolante saggio di Mariagrazia Margarito dal titolo, emblematico, Musique et parole(s) en tête à tête: sulla scia delle considerazioni della Margarito potremmo definire il monologo interiore una struttura linguistica connotante «le vague insaisissable», più precisamente «non pas un produit fini, ni même une production en train de se perfectionner», ma «le dialogue originel» - il dialogo senza voce, tra sé e sé, di Daniel Prince -, vale a dire «le préverbal, la pré-oralité inscrits sur toile de fond de silence» (in Du Dialogue au Polylogue, Acte du 3e Colloque International Do.Ri.F - Università, Rome, 24-25 octobre 1997 , textes réunis par Francesca Cabasino, Roma, Cisu, 1998, p. 218). 